\title{
Salmonella Typhimurium L Forms Induce Vascular Endothelial Cell Apoptosis via Mitochondrial- Dependent Pathway
}

Jinhai Zhai

Jiangsu Vocational College of Medicine

Tao Zhang

Bengbu Medical College

Cuiping Yang

Ruijin Hospital

Dengyu Chen ( $\square$ chengong131@sina.com )

Bengbu Medical College

\section{Research Article}

Keywords: Salmonella typhimurium, bacterial L forms, endothelial cell, apoptosis, Caspase-9

Posted Date: December 2nd, 2020

DOI: https://doi.org/10.21203/rs.3.rs-109267/v1

License: (c) (i) This work is licensed under a Creative Commons Attribution 4.0 International License.

Read Full License 


\section{Abstract}

Background Salmonella typhimurium is a pathogenic gram-negative bacterium, which is found primarily in the intestinal lumen. It often causes diarrhea in infants and young children and leads to food poisoning, as well as septicemia and septic shock. In this study, we investigated the phenomenon and mechanism of vascular endothelial cells apoptosis induced by Salmonella typhimurium $L$ forms, in order to recognize and control Salmonella typhimurium L-form infection.

Methods The apoptosis of vascular endothelial cells at 8 hours after infection with Salmonella typhimurium $L$ forms was determined by flow cytometric assay and fluoroscopy of Annexin V-FITC/PI staining. Caspase- 9 was detected by spectrophotometer.

Results Salmonella typhimurium $L$ forms can induce apoptosis of vascular endothelial cells, with significant difference in the apoptosis rate compared with the control. Caspase-9 expression is higher than that of the control.

Conclusion The ability to induce cell apoptosis of vascular endothelial cells by Salmonella typhimurium $\mathrm{L}$ forms may be related to mitochondria apoptosis pathway depending on Caspase-9.

\section{Introduction}

Vascular endothelial cells, lining the inner wall of blood vessels, are the main structure that constitutes the vascular permeability barrier and play an important role in the body's blood circulation and immune defense [1]. In this experiment, Salmonella typhimurium L forms were used to stimulate human vascular endothelial cells cultured in vitro. The Annexin V-FITC/PI double staining fluorescence method and flow cytometry were used to detect cell apoptosis, and spectrophotometric detection of Caspase-9 activation is cultivated. We explored the mechanism of vascular endothelial cell damage during Salmonella typhimurium L-form sepsis, in order to guide clinical infection prevention and treatment of Salmonella typhimurium $L$ forms.

\section{Materials And Methods}

\subsection{Bacterial Strain}

Salmonella typhimurium Standard strain CMCC 50115 was purchased from the National Institute for the Control of Pharmaceutical and Biological Products. Using the flat paper method, piperacillin drug paper was used to induce Salmonella typhimurium into L forms [2]. Subculture to obtain fresh culture of Salmonella typhimurium $\mathrm{L}$ forms, and use sterile $3 \% \mathrm{NaCl}$ hypertonic saline to make $10^{8} \mathrm{CFU} / \mathrm{ml}$ bacterial solution. Counting adopts McDonald's turbidimetric method.

\subsection{Cell line}


According to the reference methods $[3,4]$, Human umbilical vein endothelial cells were used as vascular endothelial cells in the experiment.

\subsection{Main reagents}

Annexin V-FITC/PI double staining cell apoptosis detection kit was purchased from Nanjing KaiGi Bioengineering Co., Ltd.; Caspase-9 activity detection kit was purchased from Shanghai Beibo Biological Company.

\subsection{Main instruments}

Fluorescence inverted microscope, Nikon, Japan; flow cytometer, BD company; microplate reader, Bio-Rad Model 550.

\subsection{Cell culture}

Place the vascular endothelial cells in fresh RPMI-1640+10\% FBS culture medium, and place them in a $37^{\circ} \mathrm{C}, 5 \% \mathrm{CO}_{2}$ incubator, and harvest the cells at a concentration of $10^{5} / \mathrm{ml}$. Inoculate a 6 -well cell culture plate with $2 \mathrm{ml}$ cell suspension per well. After 12 hours of regular culture, discard the old culture medium and replace it with fresh RPMI-1640 culture medium without calf serum for testing.

\subsection{Experimental grouping and induction}

The experimental groups are as follows: Group A: L-form induction group, $100 \mu$ l of Salmonella typhimurium $L$ forms bacterial solution made by adding sterile hypertonic saline to each hole of the cell culture plate; group B : Normal saline control group, adding $100 \mu \mathrm{l}$ of sterile normal saline to the control well. The cells of each group were collected 8 hours after the inducing factors were added, and the Annexin V-FITC/PI double staining flow cytometry was performed to detect the apoptosis of vascular endothelial cells induced by Salmonella typhimurium L-form, and the level of Caspase- 9 activation was detected by spectrophotometry. Make another cell slide, pre-place clean sterile small slides in each well of the cell culture plate, and then perform the experiment. After 8 hours of induction, the cell slides were taken out for Annexin V-FITC/PI double staining fluorescence microscopy.

\subsection{Apoptosis detection}

\subsubsection{Annexin V staining flow cytometry}

AnnexinV-FITC/PI double staining flow cytometry was used to detect apoptotic cells. Collect the cell suspension and operate according to the instructions. After the cells are double-stained with AnnexinVFITC/PI, they are washed twice with PBS, and tested and analyzed on the machine.

\subsubsection{Direct fluorescence method}


Use AnnexinV-FITC/PI double stain fluorescence method to detect apoptotic cells. Take cell slides, double stain with AnnexinV-FITC/PI, wash twice with PBS, observe and take pictures with inverted fluorescence microscope.

\subsubsection{Spectrophotometry}

Caspase-9 activity detection kit is based on Caspase-9 which can catalyze the substrate Ac-LEHD-pNA to produce yellow pNA. The activity of Caspase- 9 can be detected by measuring the absorbance of the reaction solution. Operate according to the kit instructions, collect the cell suspension, lyse the cells to collect the protein, add detection reagents, measure the absorbance value of A405 with a microplate reader, and subtract the absorbance value of A405 of the blank control without pNA to calculate the actual absorbance produced by pNA, Then to calculate the ratio of the experimental group and the control group to determine the activity of Caspase-9.

1.8 Statistical methods to use $t$ test and SPSS10.0 statistical analysis software for data processing.

\section{Results}

\subsection{Annexin V-FITC/PI double staining flow cytometry to detect apoptosis results}

Seeing Figure 1a, Salmonella typhimurium L forms are Gram stain negative and filamentous .Seeing Figure 1b, in the lower right quadrant of the scatter diagram, only Annexin V-FITC stained cells are apoptotic. In the experimental group Salmonella typhimurium $L$ forms can induce vascular vascular endothelial cell apoptosis. Seeing Figure 2, the apoptosis rate was higher than that of the control group, and the result was statistically significant $P<0.05$.

\subsection{Cell apoptosis results detected by Annexin V-FITC/PI double staining fluorescence method}

Figure 3, apoptotic cells only cell membrane stained green, no staining of the nucleus; necrotic cell nucleus stained red, cell membrane stained green; in Salmonella typhimurium $L$ forms group, it can be seen that there are more apoptotic cells than the control group.

\subsection{The results of spectrophotometric detection of Caspase-9 activity}

As shown in Figure 4, the degree of Caspase-9 activation in the experimental group was higher than that in the control group, and the ratio of the two activities was 1.9. The result was statistically significant, $P<0.05$.

\section{Discussion}

This experiment studies the effect of Salmonella typhimurium $L$ forms vascular endothelial cells cultured in vitro, and explores the mechanism of bacterial damage to vascular endothelial cells when Salmonella typhimurium $\mathrm{L}$ forms cause sepsis and septic shock. Vascular endothelial cells lining the inner wall of 
blood vessels are the main structure that constitutes the vascular permeability barrier, participate in many physiological and pathological processes in the body, and play an important role in the coagulation process and immune defense [5,6]. Bacteria can induce apoptosis of vascular endothelial cells through toxins, leading to the loss and shedding of endothelial cells, causing vascular endothelial damage to increase the permeability of the vascular barrier and the dysfunction of endothelial cells, and induce microcirculation disorders to cause diffuse intravascular coagulation and septic shock. Salmonella typhimurium $L$ forms are a common form of the bacterium in the infected host[7,8], and it can also cause sepsis. Whether it can induce apoptosis of vascular endothelial cells and its mechanism is the focus of this research. The prevention and treatment of the disease is of great significance.

Apoptosis is different from the cell death form of necrosis. It is regulated by the genetic material in the cell and the extracellular microenvironment. It is involved in regulating the renewal of normal cells and the removal of abnormal cells in the body under physiological conditions. It is also related to multiple systems under pathological conditions. The occurrence and development of diseases are closely related. In normal cells, phosphatidylserine is only distributed inside the lipid bilayer of the cell membrane. In the early stage of apoptosis, the phosphatidylserine in the cell membrane turns from the inside of the lipid membrane to the outside. Annexin $\mathrm{V}$ has a high affinity for phosphatidylserine and can bind to the cell membrane of cells in the early stage of apoptosis through the phosphatidylserine exposed on the outside of the cell. The Annex in V is labeled with fluorescein, and the occurrence of apoptosis can be detected by flow cytometry or fluorescence microscope. Propidium iodide $\mathrm{PI}$ is a nucleic acid dye and cannot penetrate the complete cell membrane. However, PI can penetrate the cell membrane to stain the nucleus red for cells in the middle and late stages of apoptosis and necrotic cells with incomplete cell membranes. The combination of Annexin $\mathrm{V}$ and PI can accurately detect apoptotic cells with intact cell membranes.

In the experiment, Salmonella typhimurium $L$ forms were co-cultured with vascular endothelial cells, and the apoptosis of vascular endothelial cells was detected by fluorescence microscope and flow cytometer after Annexin V-FITC/PI double staining. Caspase-9 activation was detected. When Salmonella typhimurium $L$ forms was co-cultured with vascular endothelial cells, it can induce apoptosis, due to the cells producing a large amount of reactive oxygen species and the cells themselves cannot tolerate $[9,10,11]$. Bacterial endotoxin can activate vascular endothelial cells to induce high-efficiency expression of NO synthase to produce NO and induce cell apoptosis. NO-mediated apoptosis is related to the induced expression of p53 and Bax genes. p53 regulates the transcription of Bcl-2 related pro-apoptotic gene Bax. Increased expression of Bax can form pores across the outer mitochondrial membrane, leading to a decrease in membrane potential and cytochrome $C$ As well as the outflow of apoptosis-inducing factors, cytochrome $\mathrm{C}$ forms an apoptotic complex with Apaf- 1 and pro-Caspase- 9 and causes the activation of Caspase-9 to cause apoptosis in the mitochondrial pathway [12]. The Salmonella typhimurium $L$ forms also produce toxins such as endotoxin, but the content is less than that of the original bacteria, so the virulence inducing apoptosis is weaker than that of the original bacteria [13]. 
In conclusion, the pathogenic mechanism of $L$ form injury of vascular endothelial cells caused by Salmonella typhimurium may be related to the induction of vascular endothelial cell apoptosis. Apoptosis of vascular endothelial cells leads to enlargement of endothelial fissures and increased vascular permeability. Abnormal intravascular coagulation can cause circulatory disorders and aggravate infections[14,15,16]. We look forward to the in-depth study of the damage mechanism, which will help prevent and treat infectious diseases caused by the bacteria.

\section{Declarations}

\section{Acknowledgments}

We would like to thank Anhui Key Laboratory of Infection and Immunity, Bengbu Medical College, China.

\section{Funding}

This work was supported by grants from the Key Fund Project of Anhui University Natural Science Research (KJ2016A461) and the Scientific Research Innovation Team Project of Anhui Colleges and Universities (2016-40).

\section{Availability of data and materials}

The datasets used and/or analyzed during the current study available from the corresponding author on reasonable request.

\section{Authors' contributions}

Z-JH and C-DY conceived the idea and designed the study protocol. Y-CP and Z-T contributed with provision of study material. C-DY and Y-CP conducted experiments, collected, analysed the data; C-DY and Z-T performed statistical analysis, wrote the manuscript. All authors read and approved the final manuscript.

\section{Competing interests}

The authors declare that they have no competing interests.

\section{References}

1. Watson EC, Grant ZL, Coultas L. Endothelial cell apoptosis in angiogenesis and vessel regression.Cell Mol Life Sci. 2017 Dec;74(24):4387-4403.

2. Yang C, Li H, Zhang T, Chu Y, Zuo J, Chen D. Study on antibiotic susceptibility of Salmonella typhimurium $L$ forms to the third and forth generation cephalosporins. Sci Rep. 2020 Feb 20;10(1):3042. 
3. Zhao J, Xu S, Song F, Nian L, Zhou X, Wang S. 2,3,5,4'-tetrahydroxystilbene-2-O- $\beta$-D-glucoside protects human umbilical vein endothelial cells against lysophosphatidylcholine-induced apoptosis by upregulating superoxide dismutase and glutathione peroxidase. IUBMB Life. 2014 Oct;66(10):711-22.

4. Cai M, Li S, Shuai Y, Li J, Tan J, Zeng Q. Genome-wide CRISPR-Cas9 viability screen reveals genes involved in TNF-a-induced apoptosis of human umbilical vein endothelial cells. J Cell Physiol. 2019 Jun;234(6):9184-9193.

5. Hou X, Yang S, Yin J. Blocking the REDD1/TXNIP axis ameliorates LPS-induced vascular endothelial cell injury through repressing oxidative stress and apoptosis. Am J Physiol Cell Physiol. 2019 Jan 1;316(1):C104-C110.

6. Fan X, Wang E, He J, Zhang L, Zeng X, Gui Y, Sun Q, Song Y, Yuan H. Ligustrazine Protects Homocysteine-Induced Apoptosis in Human Umbilical Vein Endothelial Cells by Modulating Mitochondrial Dysfunction. J Cardiovasc Transl Res. 2019 Dec;12(6):591-599.

7. Errington J, Mickiewicz K, Kawai Y, Wu LJ. L-form bacteria, chronic diseases and the origins of life. Philos. Trans. R. Soc. Lond. B. Biol. Sci. 2016;371:20150494.

8. Markova ND. L-form bacteria cohabitants in human blood: significance for health and diseases. Discov Med. 2017 May;23(128):305-313.

9. Kawai Y, et al. Cell Growth of Wall-Free L-Form Bacteria Is Limited by Oxidative Damage. Curr Biol. 2015;25:1613-1618.

10. Pang N, Chen T, Deng X, Chen N, Li R, Ren M, Li Y, Luo M, Hao H, Wu J, Wang L. Polydatin Prevents Methylglyoxal-Induced Apoptosis through Reducing Oxidative Stress and Improving Mitochondrial Function in Human Umbilical Vein Endothelial Cells. Oxid Med Cell Longev. 2017;2017:7180943.

11. Lu Y, Wang RH, Guo BB, Jia YP. Quercetin inhibits angiotensin II induced apoptosis via mitochondrial pathway in human umbilical vein endothelial cells. Eur Rev Med Pharmacol Sci. 2016 Apr;20(8):1609-16.

12. Zhou YQ, Zhao YT, Zhao XY, Liang C, Xu YW, Li L, Liu Y, Yang HB. Hyperoside Suppresses Lipopolysaccharide- induced Inflammation and Apoptosis in Human Umbilical Vein Endothelial Cells. Curr Med Sci. 2018 Apr; 38(2):222-228.

13. Kawai Y, Mickiewicz K, Erringto J. Lysozyme Counteracts $\beta$-Lactam Antibiotics by Promoting the Emergence of L-Form Bacteria. Cell. 2018;172:1038-1049.

14. Cui W, Leng B, Liu W, Wang G. Suppression of Apoptosis in Human Umbilical Vein Endothelial Cells (HUVECs) by Klotho Protein is Associated with Reduced Endoplasmic Reticulum Oxidative Stress and Activation of the PI3K/AKT Pathway. Med Sci Monit. 2018 Nov 24;24:8489-8499.

15. Wang X, Zhao Z, Zhu K, Bao R, Meng Y, Bian J, Wan X, Yang T. Effects of CXCL4/CXCR3 on the lipopolysaccharide-induced injury in human umbilical vein endothelial cells. J Cell Physiol. 2019 Dec; 234(12):22378-22385.

16. Yang Y, Tian T, Wang Y, Li Z, Xing K, Tian G. SIRT6 protects vascular endothelial cells from angiotensin Il-induced apoptosis and oxidative stress by promoting the activation of Nrf2/ARE signaling.Eur J Pharmacol. 2019 Sep 15;859:172516. 
Figures

a

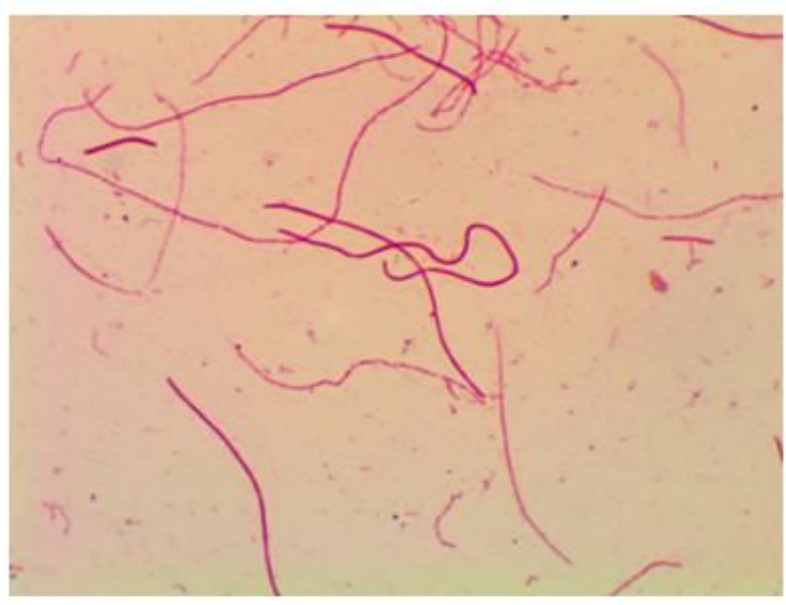

b

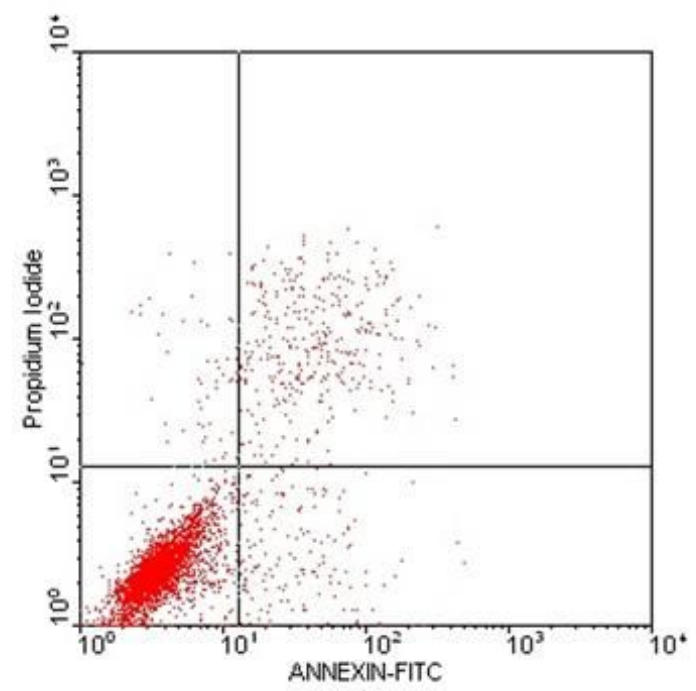

\section{Figure 1}

Salmonella typhimurium $L$ forms staining and apoptosis flow cytogram Figure 1a Salmonella typhimurium L forms (Gram stain, magnification: 1000x); Figure 1b Scatter plot of apoptosis detection by flow cytometry. 
a

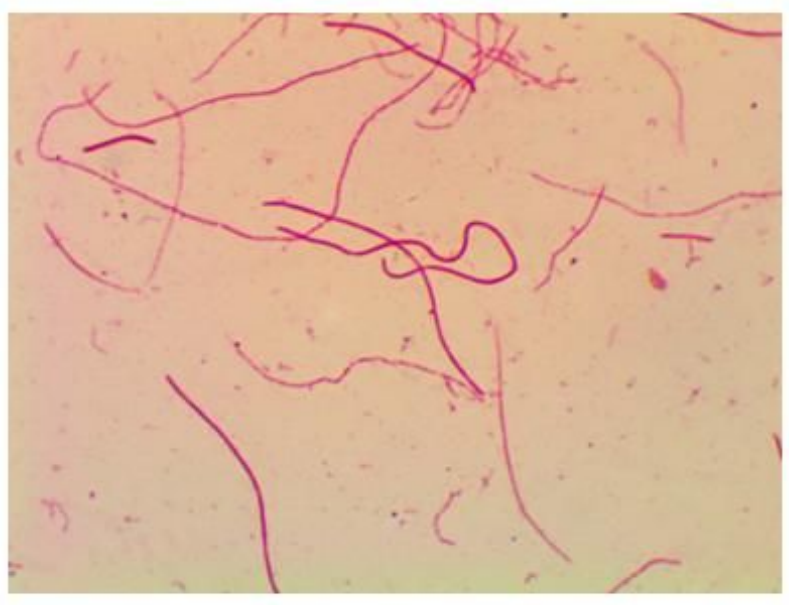

b

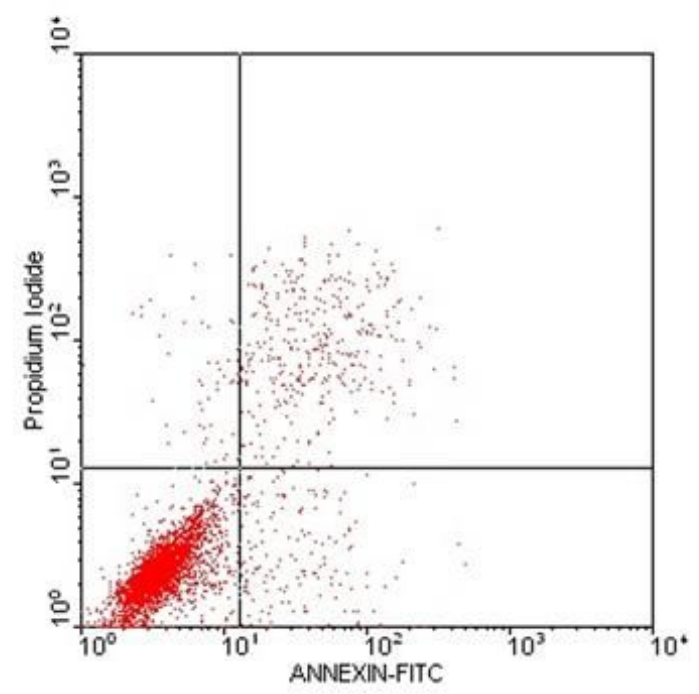

\section{Figure 1}

Salmonella typhimurium $L$ forms staining and apoptosis flow cytogram Figure 1a Salmonella typhimurium L forms (Gram stain, magnification: 1000x); Figure 1b Scatter plot of apoptosis detection by flow cytometry. 


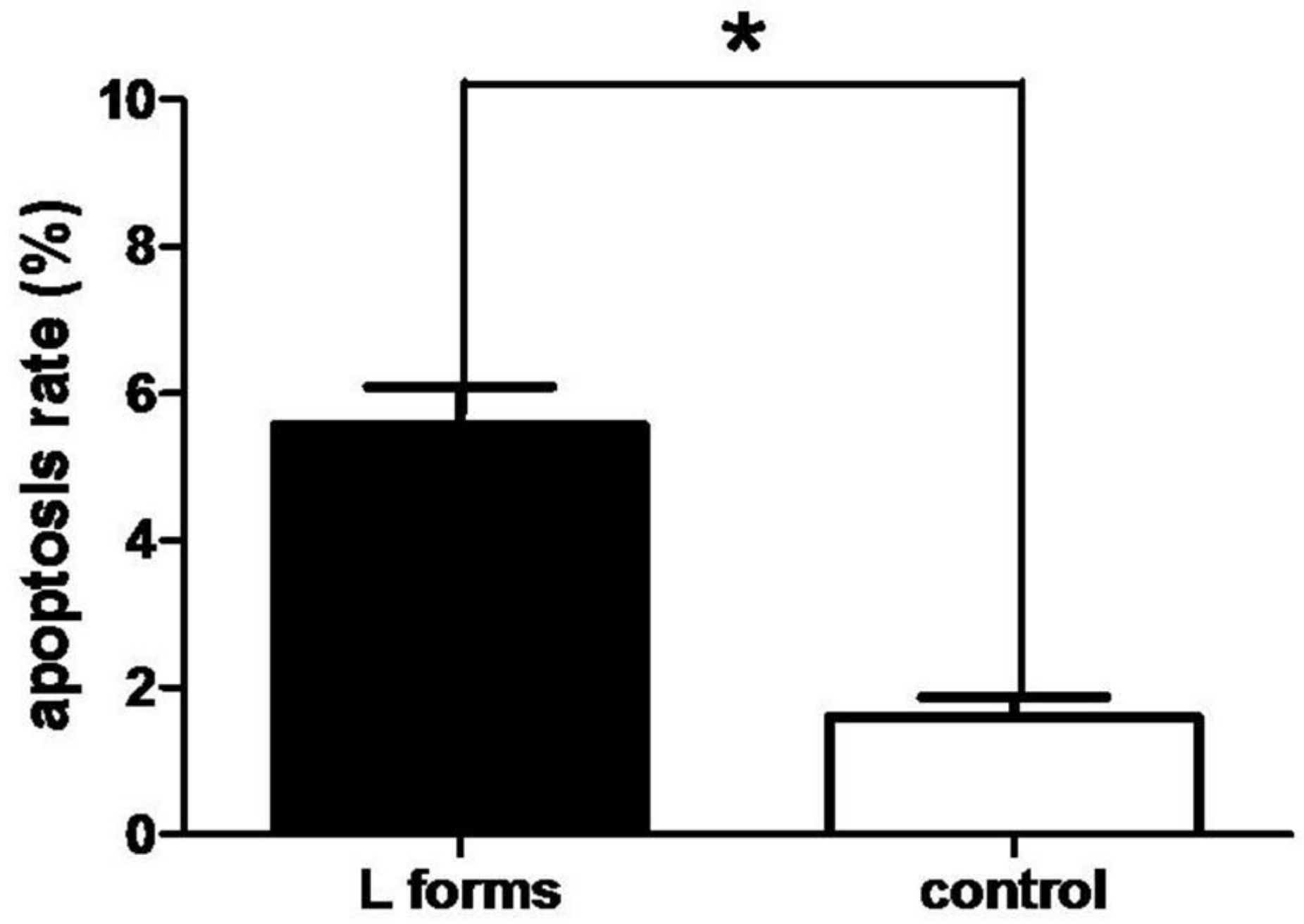

Figure 2

Flow cytometry analysis of cell apoptosis rate Note: Compared with the control group, * $\mathrm{P}<0.05$ 


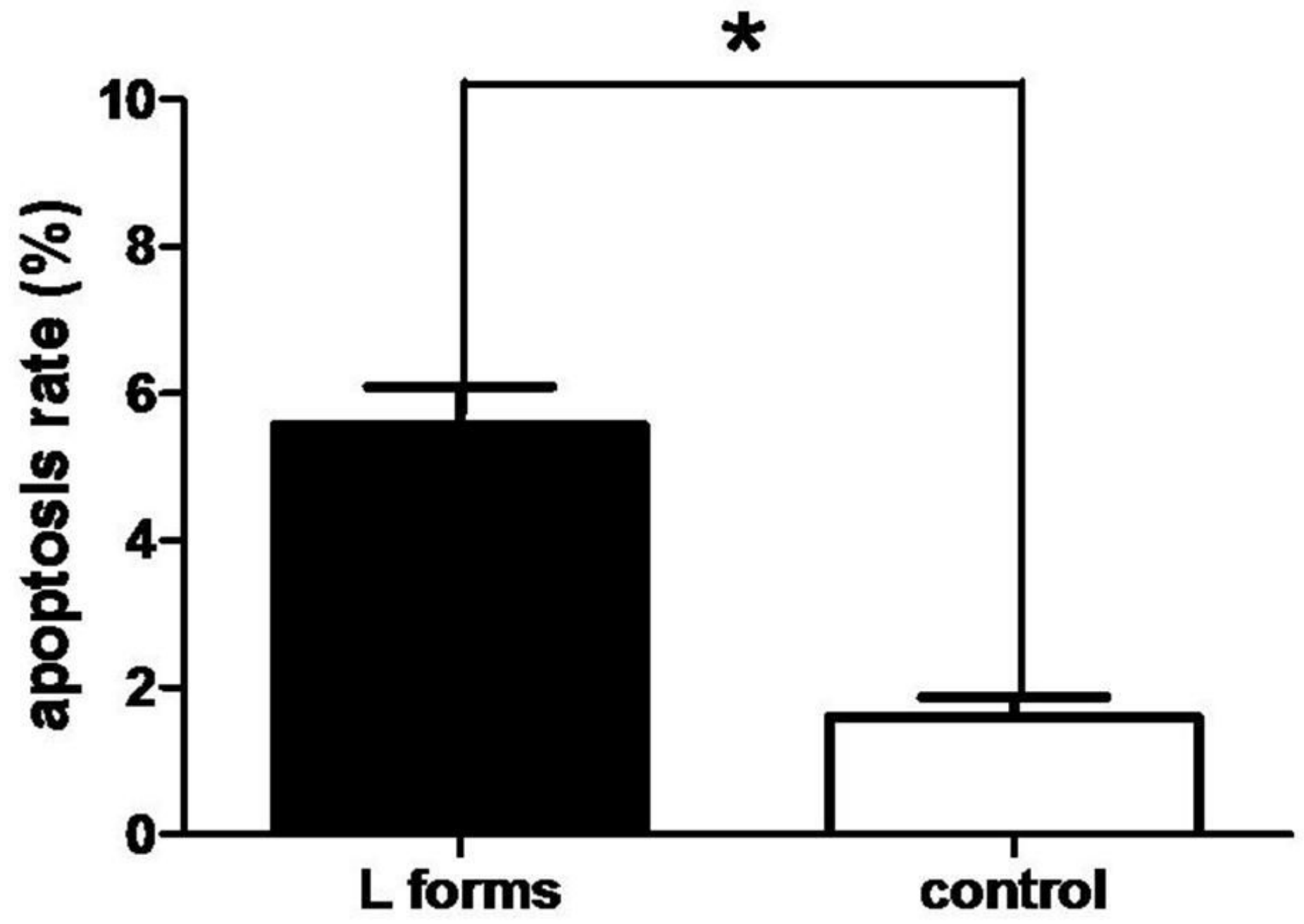

Figure 2

Flow cytometry analysis of cell apoptosis rate Note: Compared with the control group, * $\mathrm{P}<0.05$ 


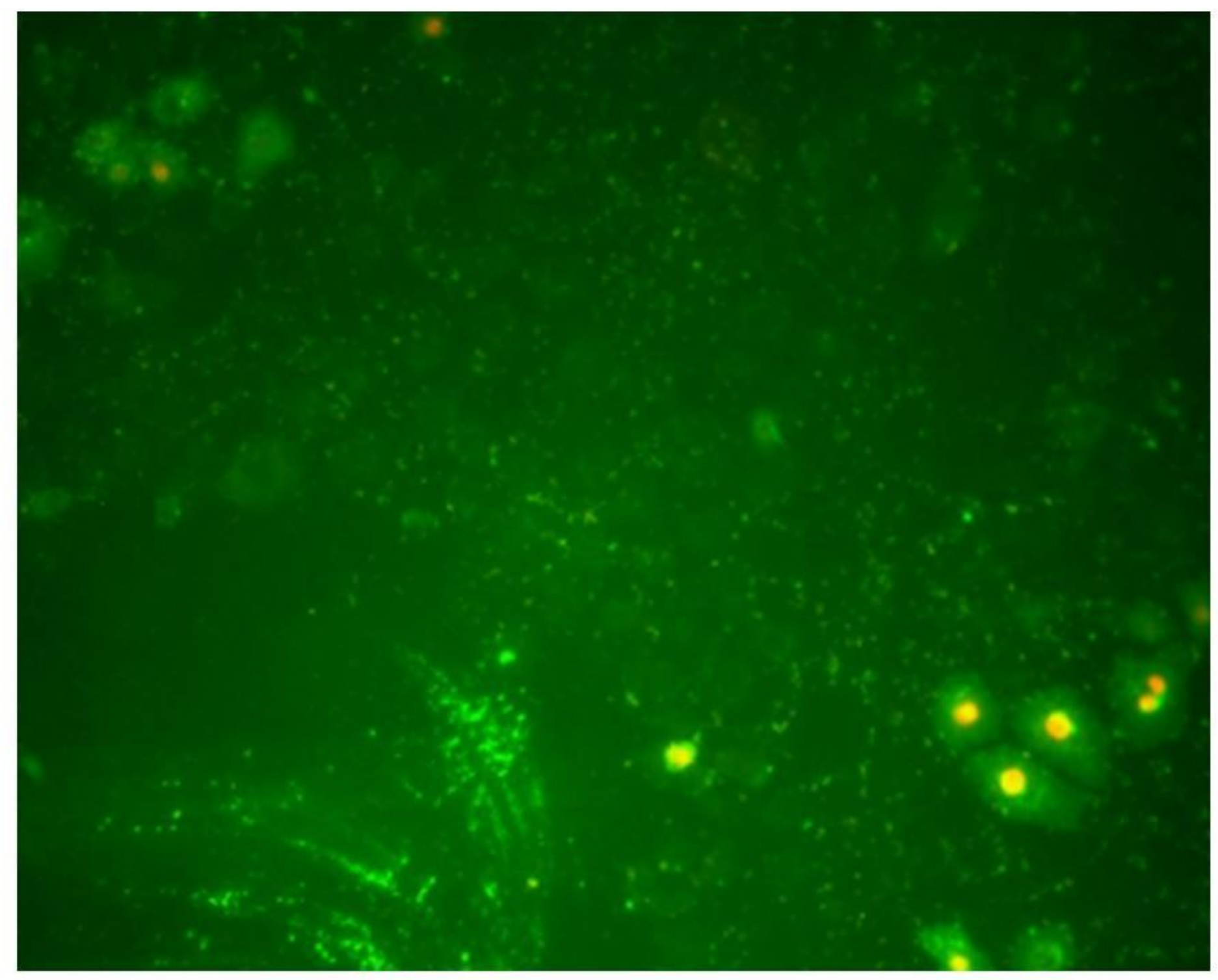

Figure 3

Detection of apoptotic cells by fluorescence microscopy 


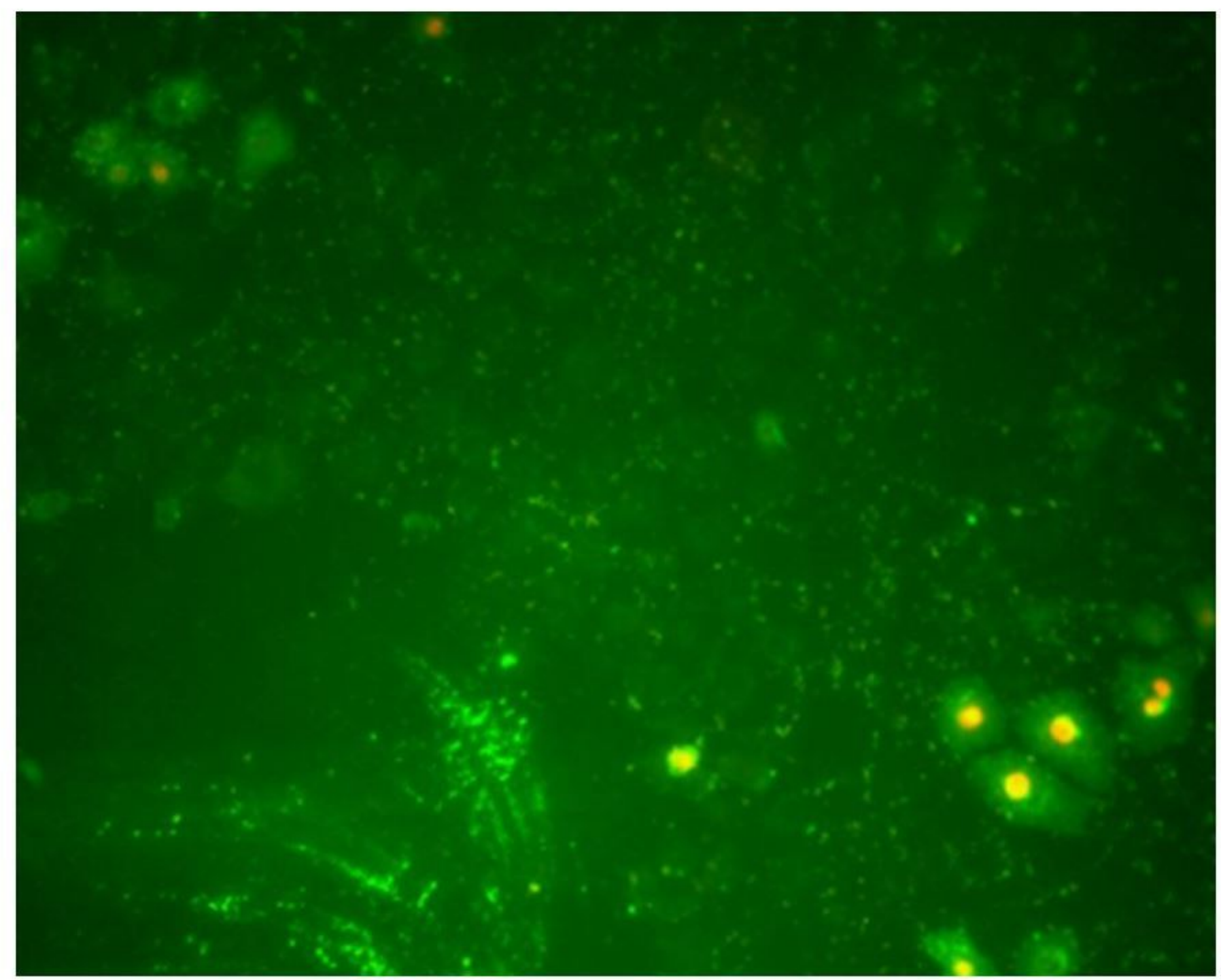

Figure 3

Detection of apoptotic cells by fluorescence microscopy 


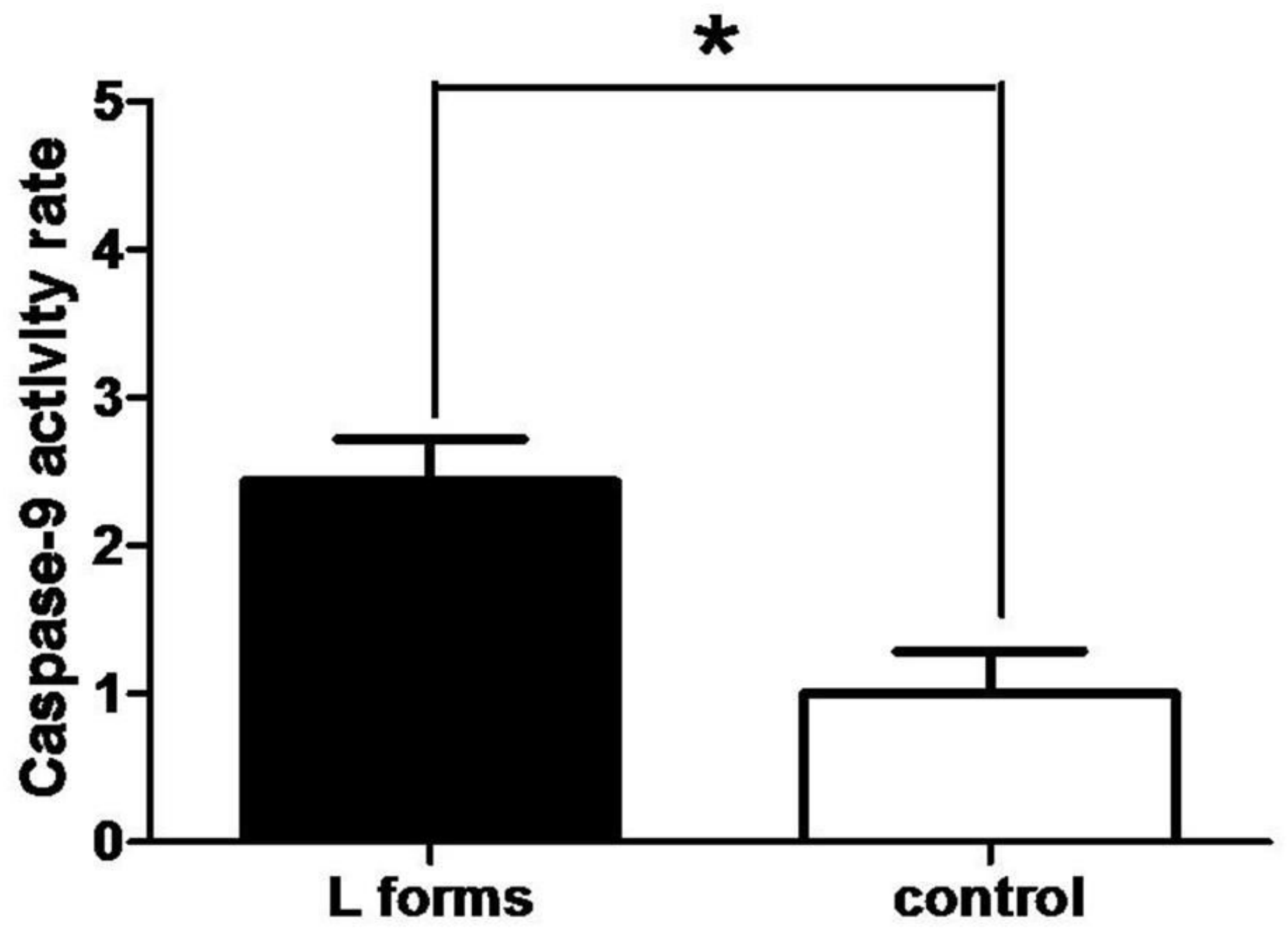

Figure 4

Detection of Caspase- 9 activity by spectrophotometry Note: Compared with the control group, * $\mathrm{P}<0.05$ 


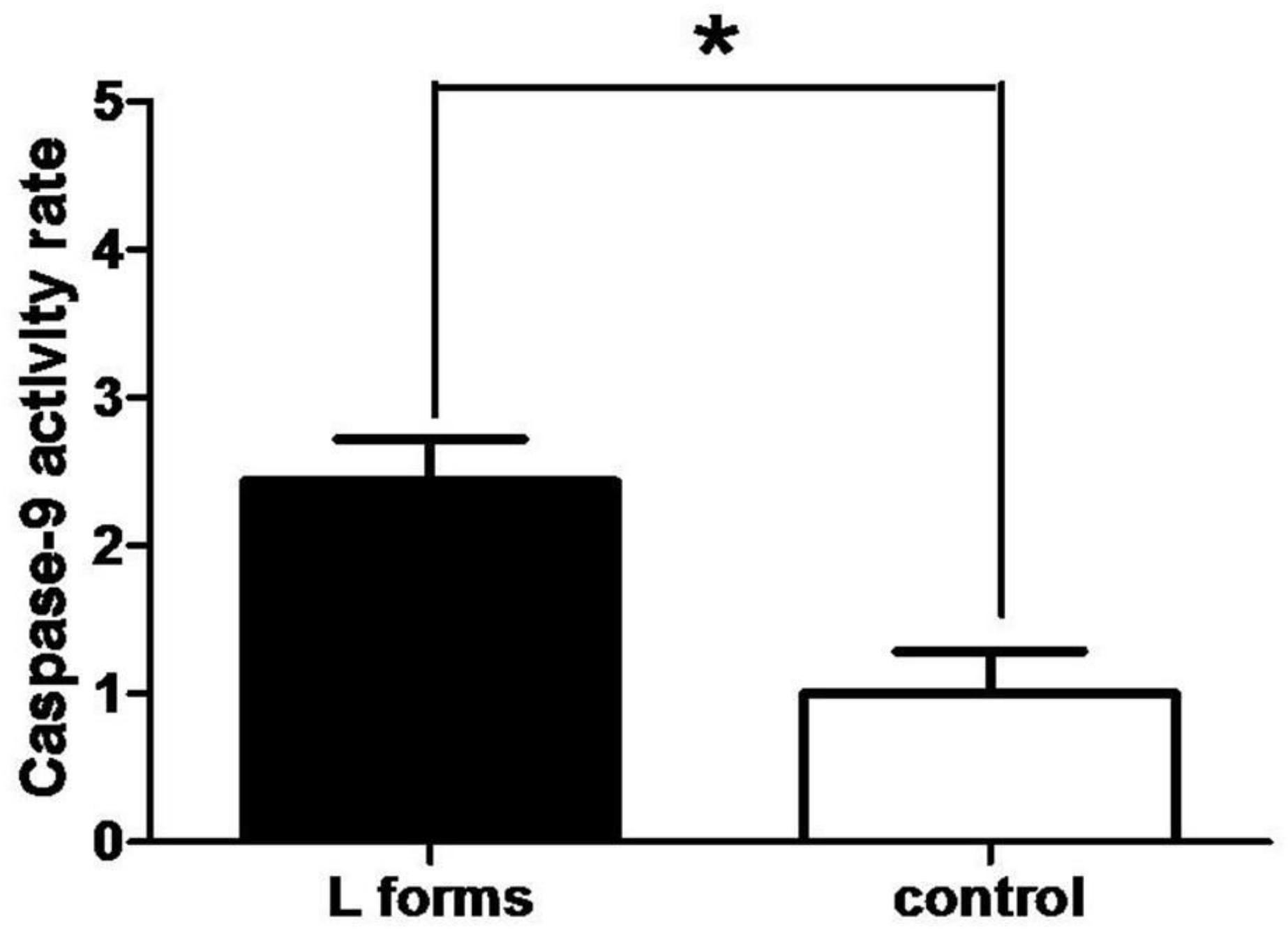

Figure 4

Detection of Caspase- 9 activity by spectrophotometry Note: Compared with the control group, * $\mathrm{P}<0.05$ 